\title{
3D Packaging Architecture Using Paper as a Dielectric Medium
}

\author{
Amin Rida*, Li Yang, Napol Chaisilwattana, Scott Travis, Swapan Bhattacharya and Manos M. Tentzeris \\ RFID/Sensor Lab, Georgia Electronic Design center, \\ School of Electrical and Computer Engineering \\ Georgia Institute of Technology, Atlanta, GA 30332-0250 \\ *E-mail: arida@ece.mail.gatech.edu
}

\begin{abstract}
Radio Frequency Identification (RFID) can be utilized for the tagging of a wide variety of application. A Planar Inverted F Antenna (PIFA) for real time locating has been designed, built and characterized on paper substrate. In specific the PIFA for tagging of metallic objects such as containers in ports or automotive applications is designed, fabricated, and characterized on a paper substrate. The antenna interconnect, or simply the shorting via A via, was drilled though the paper substrate using laser in one of the corners of the structure and, and is characterized by plotting $\mathrm{S}$ parameters. Agreement between simulation and measurements provide the proof of concept that paper is a good candidate for a multilayer low cost substrate. This effort proves for the first time, the feasibility of building 3D structure on paper with zinterconnects.
\end{abstract}

\section{Introduction}

RFID is a wireless automatic identification method that uses the electromagnetic (EM) waves as a mean of communicating data between an RFID reader and an RFID tag placed on an object. RFID which does not require Line of Sight (LoS) to communicate with the reader and allows for simultaneous read/write from multiple tags, can modify or erase information already defined in tags such as the WRITE or KILL command, as well as an easy remote and selective activation of sensor devices based on their unique ID's [1].

Due to increasing demand for automatic identification, RFID's find countless applications in different areas such as item-level tracking, access control, electronic toll collection and vehicle security. However, conventional or generally used label-fabricated RFID tags do not operate when in close vicinity to metals since the EM wave becomes totally reflected by metallic surfaces in a destructive manner unless placed at a certain interval (such as multiple of $\lambda / 4$; where $\lambda$ is the wavelength of the EM wave, which is not feasible in many tracking applications), and while metallic objects such as cargo containers in ports, building construction parts such as pipes, Automotive-ID, Aero-ID, and space witness more demand for automatic identification, an RFID enabled on metal mount becomes crucial.

On the material level, this places a vast amount of demand on the importance on the substrate/package. Paper is considered as one of the best organic substrates for RFID tags. First of all, paper is environmentally friendly and can undergo large reel to reel processing. In addition, paper is compatible with circuit printing by direct write methodologies. This is one of the biggest advantages of paper since active tags require additional modules like sensors and batteries to be mounted or embedded. A fast ink jet printing process can be used efficiently to print these modules on or into the paper substrate. Paper can also host nano-scale additives (i.e. fire retardant textiles) and can be made hydrophobic. Most importantly its dielectric constant $\varepsilon_{\mathrm{r}}(\sim 3)$ is close to that of air (5-6\% power reflection), therefore, electromagnetic power can still penetrate easily even if the RFID is embedded in the substrate. The dielectric characterization has been achieved by the authors for the UHF frequency range [2]. The results of dielectric constant and dielectric loss tangent have been used in the antenna design sections. In this paper, and for the first time, the characterization of a shorting via or z-interconnects has been recorded through the S-parameter characterization of a PIFA Antenna.

\section{PIFA Design}

The fast progress in wireless communications, such as video services, mobile TV and Radio Frequency Identification, requires the development of high-density microwave packaging. On the antenna side, the development of small integrated antennas becomes a must to ensure meeting the rapidly expanding commercial communication applications.

Planar inverted-F antennas (PIFA) have been widely accepted for portable handset applications, due to its low profile nature and the ability to realize dual/multi band operation. It also only requires simple manufacturing, since the radiating structure can be printed. A paper-based PIFA antenna is designed and simulated in this section, showing the effect of the realized via structure for the first time on paperbased substrate.

The PIFA consists of four key elements, a ground plane, a top planar element, a feeding wire between the ground plane and the top, and a shorting wire which is connecting the ground plane and the top planar element. The antenna is fed at the base of the feed wire at the point where the wire connects to the ground [3]. The shorting wire allows good impedance match to be achieved, resulting a much smaller overall size of a PIFA than a traditional half-wavelength probe-fed patch antenna. While in the design, the effects of the ground plane and the different parts all need to be considered to ensure good performance.

A U-shaped slot is opened on the radiating patch. The existence of such a slot will introduce a secondary band to the PIFA structure [4]. The PIFA diagram is shown in Fig. 1 along with the dimensions of the U-shaped slot, via and 
feeding structure radii and placements. Fig. 3 shows a photograph of the PIFA after it was built using conventional copper tape. The location of the via and of the feeding point is shown in Fig 2 demonstrating the exact location of the feeding point of the antenna by a typical 50 Ohms SMA connector on the back side of the Antenna.

Without the shorting via, the antenna is a typical patch antenna, and only one resonant peak can be observed. The simulated return loss is plotted in Fig. 1 (a), with one peak at $2.25 \mathrm{GHz}$. When the via is introduced and metalized, the antenna becomes a PIFA antenna, and the secondary band shows up. The simulated return loss is plotted in Fig. 3, with one resonant peak at $0.68 \mathrm{GHz}$ and another at $2.48 \mathrm{GHz}$. The showing of the lower resonant frequency can be used to determine the successful realization of the via hole. Radiation pattern of the two resonant frequencies are also plotted in Fig. 5.

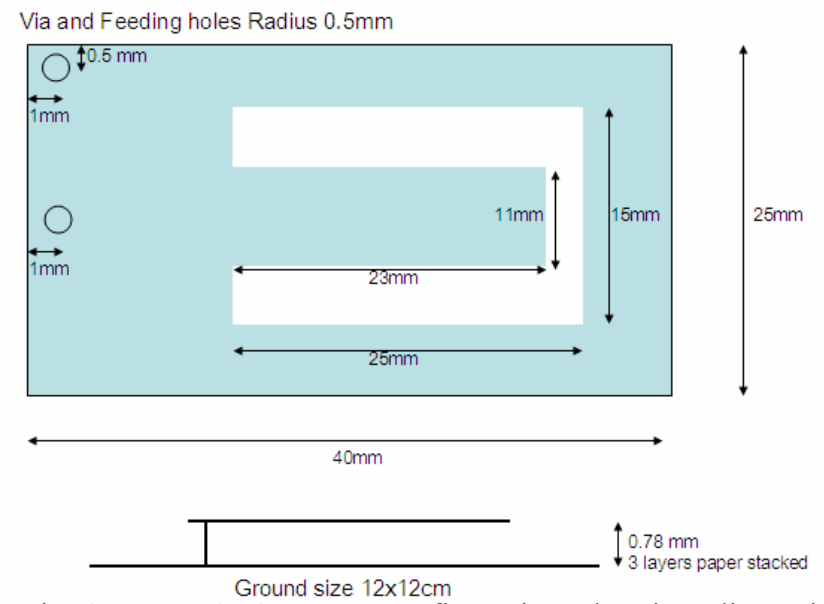

Fig 1. PIFA Antenna configuration showing dimensions (top) and cross section (bottom) of the design.

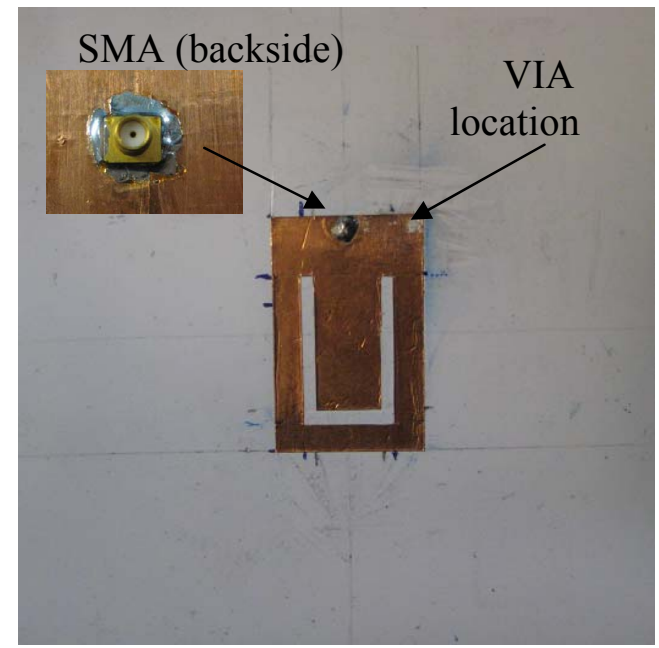

Fig 2. Photograph of PIFA showing via and feeding locations.

Measurement results were achieved using ZVA-8 VNA and are shown in Fig. 4 for the structure with VIA. These results show a good agreement with a slight shift in resonance frequency that could be due to several reasons. It is to be noted that the structure was formed by cutting copper tape by hand to the required dimensions and so significant inaccuracy might have occurred and caused this frequency shifting. Hence, measurement results show proof of concept of formation of via holes in paper substrate.

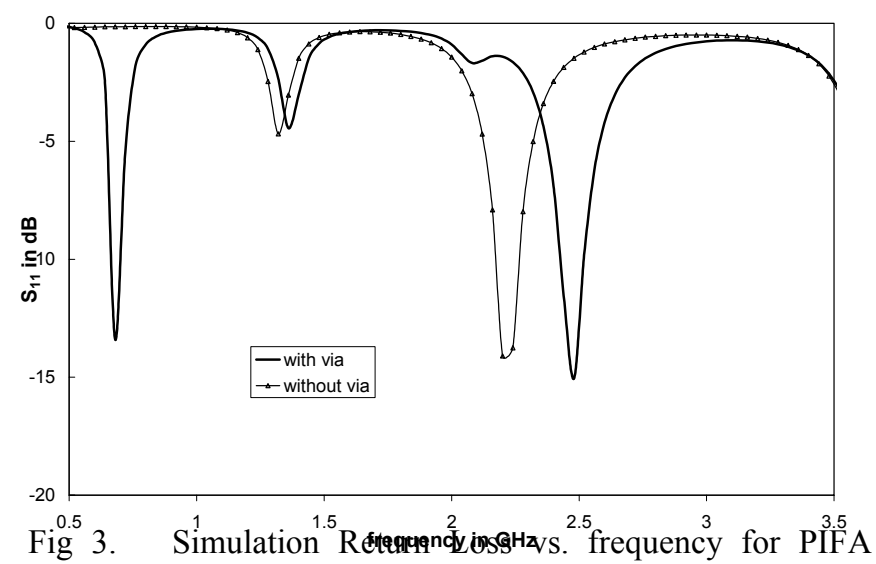
Antenna.

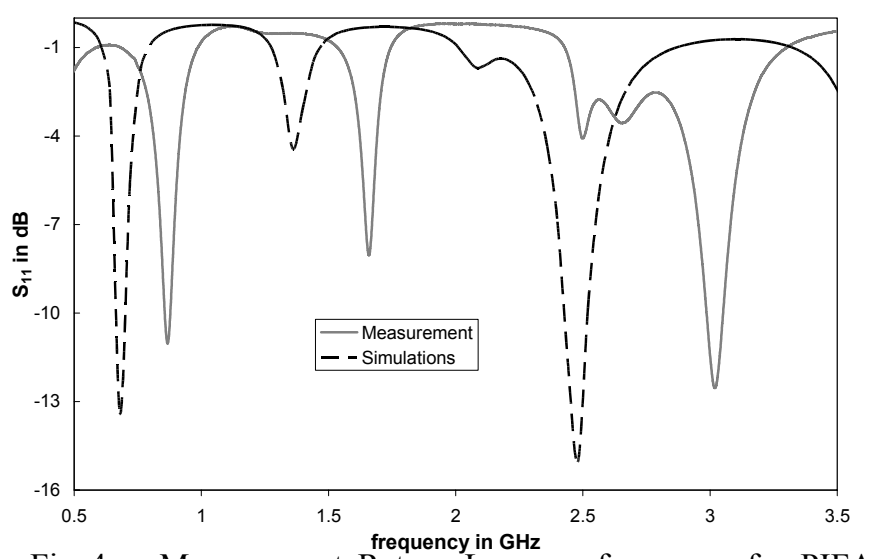

Fig 4. Measurement Return Loss vs. frequency for PIFA Antenna with VIA compared with Simulated results.

\section{VIA Formation}

Metallized vias are necessary for layer to layer registration in the vertical direction. A carbon dioxide laser having a wave-length of 10.6 micrometer was selected for via construction. Since, $\mathrm{CO} 2$ laser beam is not easily absorbed by metal, the beam can be stopped at the metal layer (ground plane) underneath the laminated paper substrate, therefore making an easy route for the via filling process. The theoretical limit of the $\mathrm{CO} 2$ laser used in this study is about 250 micrometer. Hence, the via hole had to be larger than $0.25 \mathrm{~mm}$. Moreover, the thickness of the multi-layer paper thickness was on the order of $0.75 \mathrm{~mm}$, therefore, the via opening was adjusted to $1 \mathrm{~mm}$ in diameter. Multiple (3 to 4) passes of the focused laser beam were required to drill through the paper layers. The debris inside the via hole and around the via wall were removed mechanically followed by rinsing with isopropyl alcohol. Reactive ion etching will be implemented in future for the via clearing process. 


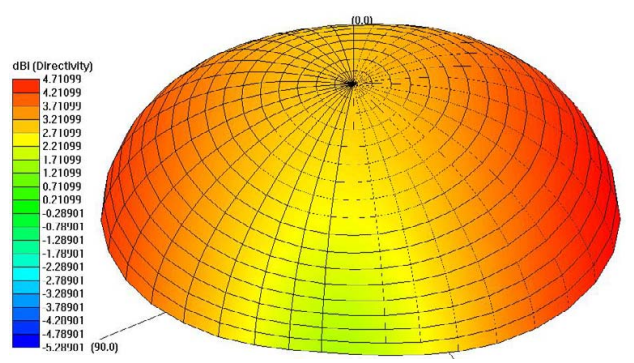

(a)

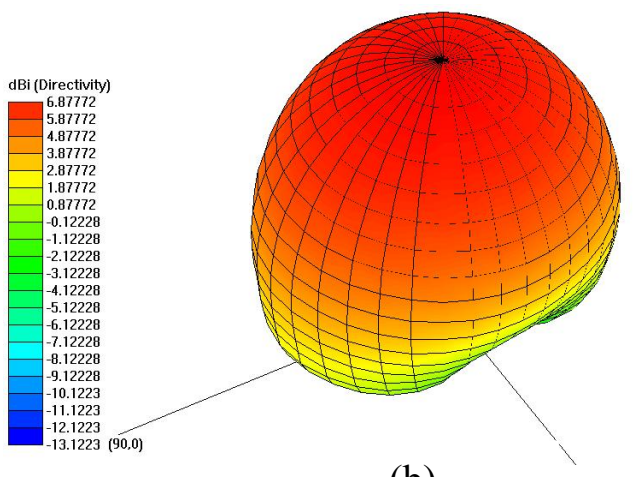

(b)

Fig. 5. Radiation Pattern at two resonant frequencies, $x$ (a) directivity $4.71 \mathrm{~dB}$ at $0.68 \mathrm{GHz}$ (b) directivity $6.87 \mathrm{~dB}$ at $2.48 \mathrm{GHz}$.

\section{IV.3D Paper-on-Paper with Embedded Components}

Conventional lamination and copper etching chemistries may be utilized for the formation of passive structures on paper substrate. This method is an operator intensive but can be easily adopted in a PCB fabrication house and therefore is more attractive toward large volume manufacturing at lower cost.

The goal is to integrate RFID components with sensors and thin film batteries for active tags with high read/write range wireless sensing in rugged industrial, warfare and automotive environments. To achieve the ultimate goal of 3D paper-on-paper packaging with embedded passives, MEMS, RFIDs, sensors, thin film batteries, and discretes and ICs, several bottlenecks needs to be overcome since none of these processes have been optimized or even addressed.

The lamination process was optimized by varying temperature, pressure, and residence time, as well as the heating and cooling rates. The copper foil was bonded without any visible delamination or voids. The photolithography process was done using a dry film photoresist followed by UV exposure and finally etching copper using a slow etching chemistry. The laminated board was then dried in an oven at $100^{\circ} \mathrm{C}$ for 30 minutes. The fabricated structures had good adherence to the paper substrate as was evident by a simple scotch tape test.

A multi-layer core has been fabricated using paper-on-paper lamination, the thickness achieved is 1 and $2 \mathrm{~mm}$ layers of ink-jet printable copper. Figure 6 shows the parallel plate capacitors fabricated on laminated multi-layer $1 \mathrm{~mm}$ thick paper substrate through copper foil lamination and etching. The current work is focused toward ink jet printing of passives structures ( $\mathrm{R}, \mathrm{L}$, and $\mathrm{C}$ ) and then laminating to form a multi-layer structure with cut out cavity for embedding active chips.

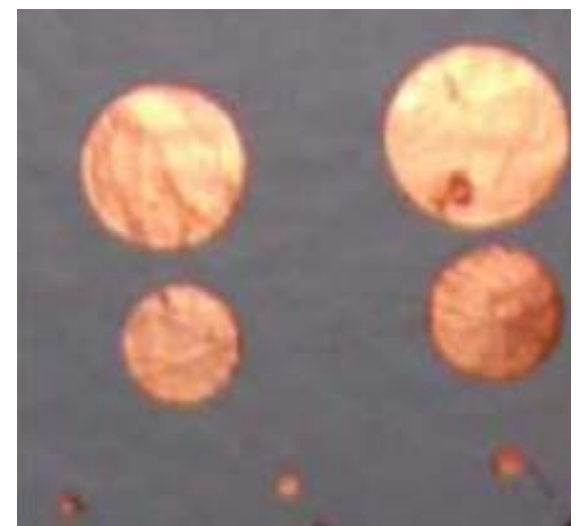

Fig 6. Fabricated parallel plate capacitors on $1 \mathrm{~mm}$ thick paper substrate.

\section{Conclusion}

A PIFA Antenna on a multilayer paper substrate and with a shorting VIA structure for real time locating has been designed, built and characterized. Agreement between simulation and measurements provide the proof of concept that paper is a good candidate for a multilayer low cost substrate. This effort proves for the first time, the feasibility of building 3D structure on paper with z-interconnects.

\section{Acknowledgments}

The authors would like to acknowledge Creative Materials for supplying the conductive adhesives.

\section{References}

[1] K. Finkenzeller, RFID Handbook, 2nd ed., Wiley, 2004.

[2] L. Yang, A. Rida, R. Vyas, M. M. Tentzeris, "RFID Tag and RF Structures on a Paper Substrate Using InkjetPrinting Technology," Microwave Theory and Techniques, IEEE Transactions on Volume 55, Issue 12, Part 2, Dec. 2007 Page(s):2894 - 2901

[3] K. Virga, Y. Rahmat-Samii, "Low-profile EnhancedBandwidth PIFA Antennas for Wireless Communications Packaging," Microwave Theory and Techniques, IEEE Trans., pp 1879-1888, vol. 45, No. 10, 1997.

[4] P. Salonen, M. Keskilammi, and M. Kivikoski, "SingleFeed Dual-Band Planar Inverted-F Antenna with Ushaped slot," Antennas and Propagation, IEEE Trans., pp. 1262-1264, vol. 48, No. 8, 2000. 